Ciencia y Educación, Vol. 4, No. 3, septiembre-diciembre, 2020

ISSN (impreso): 2613-8794・ISSN (en línea): 2613-8808

DOI: https://doi.org/10.22206/cyed.2020.v4i3.pp65-77

\title{
Profesores en la era digital: prácticas en la escuela secundaria argentina
}

\author{
The role of teachers in the digital age: practices \\ in argentine secondary school
}

Virginia Saez ${ }^{\mathrm{a}}$ ORCID: 0000-0002-3043-4614

Recibido: 14/02/2020 • Aprobado: 02/04/2020

Cómo citar: Saez, V. (2020). Profesores en la era digital: prácticas en la escuela secundaria argentina. Ciencia y Educación, 4(3), 65-77. Doi: https://doi.org/10.22206/cyed.2020.v4i3.pp65-77

\section{Resumen}

El contexto de convergencia digital demanda de la escuela nuevas alfabetizaciones. Resulta relevante el análisis de los sentidos otorgados a los medios digitales por los profesores como pilar para movilizar cambios en las estrategias de enseñanza. Esta investigación caracterizó el uso doméstico y pedagógico de la web 2.0 por parte de los profesores, se identificaron las debilidades en la formación y los sentidos que construyen sobre el uso de los medios digitales en el contexto educativo. Se realizó un estudio cualitativo en escuelas secundarias públicas argentinas. Los instrumentos de recolección fueron: observaciones de clase, entrevistas en profundidad y notas de campo. Las dimensiones que contemplan la estrategia de recolección de información son: la identificación, la formación tecnológica, el perfil informático, prácticas pedagógicas y sentidos/percepciones. Tras el análisis del corpus, se concluyó que es necesario fortalecer la educación mediática de los profesores para transformar el uso pedagógico de los medios digitales.

Palabras clave: escuela secundaria; nuevas tecnologías; formación de docentes; medios de comunicación de masas; educación mediática.

\begin{abstract}
The context of digital convergence demands new literacy from the school. The analysis of the senses granted to digital media by teachers as a pillar to mobilize changes in teaching strategies is relevant. This research characterized the domestic and pedagogical use of the web 2.0 by teachers, identified weaknesses in the training and senses they build on the use of digital media in the educational context. A qualitative study was conducted in argentine public secondary schools. The collection tools were: class observations, in-depth interviews, and fieldnotes. The dimensions of the information collection strategy are: identification, technological training, computer profiling, pedagogical practices and senses/perceptions. Following the analysis of the corpus, it was concluded that it is necessary to strengthen the media education of teachers in order to transform the pedagogical use of digital media.
\end{abstract}

Keywords: High school; teacher; new technology; teacher education; mass media; media education.

\footnotetext{
a Consejo Nacional de Investigaciones Científicas y Técnicas, del Instituto de Investigación en Ciencias de la Educación. Universidad de Buenos Aires, Argentina. Correo-e: vsaez@filo.uba.ar
} 


\section{Introducción}

El ecosistema comunicativo actual requiere ciudadanos idóneos en los medios digitales. Interpela a los profesores para que enseñen en la complejidad de conocimientos que convergen en distintos formatos y lenguajes multimodales (Martín, 2019). La evolución de las tecnologías nos ubica de cara a inéditas modalidades de circulación y producción del saber, que demanda estudiar los medios como nuevos modos de percepción y expresión.

Los medios digitales atraviesan contextos culturales, sociales y políticos que, a su vez, los reconfiguran y les dan significatividad. Una serie de investigaciones del campo de las pedagogías críticas proponen la necesidad de nuevas alfabetizaciones (Jenkins, Saad, Fung y Bosch, 2019; Dussel y Trujillo, 2018, Southwell, 2019, Tyner, 2014; Gutiérrez y Torrego, 2018), en tanto los medios tecnológicos involucran nuevos estilos de habla y escritura, prácticas discursivas con formas inéditas de apropiación, lectura y resemantizacion (Kerbrat, 1980; Ferrés y Piscitelli, 2012). Bajo la consideración de que la Educación Mediática refiere al modo de enseńar y aprender sobre los medios y las tecnologías, identificamos que es un campo interdisciplinario y sus contenidos son un objeto en disputa (Saez y Richter, 2019).

Las políticas públicas destinadas a la inclusión de los nuevos medios en los salones de clase han renovado el piso tecnológico de las escuelas. En Argentina, hubo un crecimiento exponencial ante el surgimiento del modelo 1 a 1: a nivel nacional el Programa de Inclusión Digital Educativa Conectar Igualdad (CFE en el año 2010) y en el ámbito de la CABA el Plan Sarmiento (implementado a partir del 2011).

Pero aún no se observa una transformación en las prácticas docentes. Si hay que formar a los estudiantes para vivir en la cultura digital, es necesario preparar a los educadores en esta área, no basta con la provisión de computadoras y posibilitar el acceso a internet. Sin embargo, a pesar de las inversiones realizadas a través de los distintos planes de inclusión digital, con el fin de universalizar la apropiación de las nuevas tecnologías y democratizar el acceso al conocimiento, no se ha logrado un avance en la realidad educativa. Lo relevante para las instituciones educativas no es solo tener acceso a los medios, sino la capacidad de los educadores para utilizarlos en el acto de enseñar con el fin de incrementar aprendizajes significativos.

Se estima imprescindible la preparación docente, desde la formación inicial y en la formación continua, para enfrentar estos desafíos educativos (Delgado, Casado y Lezcano, 2016). Se requiere una transformación metodológica en el trabajo de los profesores, para que los educadores logren integrar los nuevos medios y sus oportunidades pedagógicas, sin reproducir con las mismas herramientas aquello que se hacía sin ellas. Al respecto, no solo hacemos alusión a un manejo técnico de los nuevos medios, sino a formar una mirada amplia e inteligente hacia su uso y la información que brindan, con el fin de producir conocimiento y compartirlo en las redes (Gutiérrez y Torrego, 2018; Saez, 2019a).

En este sentido, resulta relevante indagar la distinción entre el uso doméstico y el uso pedagógico de los medios digitales, en función del propósito y el ámbito donde se utilizan. Con uso doméstico referimos al empleo de los medios digitales como herramientas para resolver problemas de la esfera personal, que se desenvuelve en su mayor parte en el ámbito del hogar. Por su parte, con uso pedagógico aludimos a la utilización de los medios digitales como herramientas en la práctica docente, que se desarrolla en la escuela o fuera de ella.

Ahora bien, la formación del profesorado en el uso de los medios digitales resulta un desafío en nuestra región. En Latinoamérica, la aproximación a los bienes simbólicos es muy dispar y el discurso mediático es relevante en el proceso comunicativo (Orozco, 2018). Los avances en la sociedad del siglo XXI impactan de forma directa en el sistema educativo regional, y las generaciones jóvenes son las que, en general, mantienen un vínculo más estrecho con los medios digitales y tienen mayor disposición a atender sus códigos. Sin embargo, la apropiación y la utilización de las tecnologías de la información y la comunicación es desigual. En el caso argentino, la competencia informática en los 
adolescentes entre 13 y 17 años presenta diferencias significativas de una clase social a otra (Kaplan y Piovani, 2018). La distribución y apropiación del capital informático condiciona el tipo de trayectorias sociolaborales que puedan desarrollar y conlleva consecuencias a largo plazo.

Tras el análisis de los estudios antecedentes, sostenemos como hipótesis interpretativa que la falta de educación mediática es una dimensión interviniente en la producción y reproducción de las marginaciones sociales. Específicamente el aprendizaje con los nuevos medios, es un requisito de época en la era digital para poder acceder al derecho a la educación y la comunicación.

En virtud de la dinámica versátil de la cultura digital, asumimos que resulta relevante el análisis de la inclusión de los medios digitales por parte de los docentes en el nivel secundario, así como de las disposiciones y preferencias de los educadores como pilar para movilizar un cambio en las estrategias de enseñanza en el contexto argentino.

\section{Metodología}

Un pilar para el fortalecimiento de la inclusión en el sistema educativo de los medios digitales se halla en los profesores; pero son escasos las investigaciones que analizan la educación mediática en la escuela secundaria argentina. Así, es arduo saber si están formados para enfrentar los desafíos de los cambios culturales, identificar debilidades y amenazas, y elaborar formulaciones superadoras.

En el presente estudio, nuestro objetivo es caracterizar el uso doméstico y pedagógico que los profesores realizan de la web 2.0, identificar las debilidades en la formación, y los sentidos que construyen sobre el uso de los medios digitales en el contexto educativo.

En función de las particularidades de nuestro objeto de investigación se llevó a cabo un estudio de corte cualitativo (Sirvent, Monteverde, Clerici, Peruzzo y Agulló, 2016). Para ello, se desplegaron decisiones y estrategias metodológicas consistentes con un diseño flexible.

\section{Muestra}

En función del objeto de estudio y la perspectiva metodológica descrita se desarrollaron estrategias y técnicas de relevamiento de la información para contribuir a la comprensión de nuestro objeto. El diseño para abordar el estudio fue flexible.

La demarcación de la muestra fue escalonada en función de tres decisiones: elección de las escuelas secundarias, de las asignaturas y la de los profesores. En primer lugar se seleccionaron dos instituciones en relación con el criterio de accesibilidad y predisposición al estudio por parte de los sujetos y las instituciones formadoras. Se buscaron dos escuelas secundarias que contaron con características similares, que resultan fértiles para analizar la construcción de comunicación sobre los medios digitales: son establecimientos públicos de gestión estatal del área sur de Buenos Aires y atienden a población estudiantil de sectores socialmente desfavorecidos. Este criterio resulta de relevancia ya que partimos del supuesto de que existen brechas digitales y culturales que inciden negativamente en la construcción del capital cultural de los estudiantes y es en este tipo de establecimientos donde toma especial relevancia la construcción de conocimiento sobre los medios de comunicación (Dussel y Quevedo, 2010).

Bajo estas consideraciones, a los fines de alcanzar una comprensión profunda acerca del uso de los medios digitales en la escuela secundaria, hemos realizado un extenso trabajo de campo en dos escuelas secundarias enclavadas en zonas urbanas periféricas de Buenos Aires, durante el primer cuatrimestre del año 2019.

Es relevante mencionar que la mayoría de los docentes que participaron en la investigación son: mujeres $(65 \%)$ y jóvenes $(70 \%)$, tienen entre 34 y 43 ańos. Todos los docentes entrevistados poseen el título docente habilitante del nivel, y la mayor parte $(85,5 \%)$ culminó una carrera de grado, aunque no se requiera esta titulación para ejercer en la escuela secundaria (véanse gráficos 1,2 y 3 ). 
Gráfico 1. Sexo

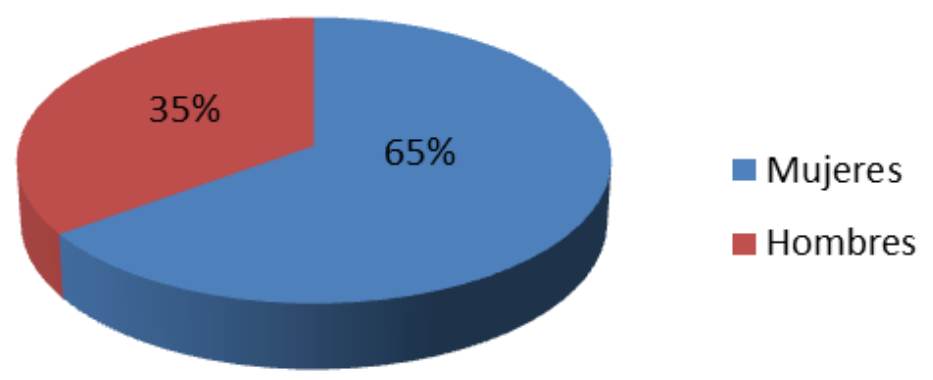

Fuente: elaboración propia a partir de los datos de la presente investigación.

Gráfico 2. Edad

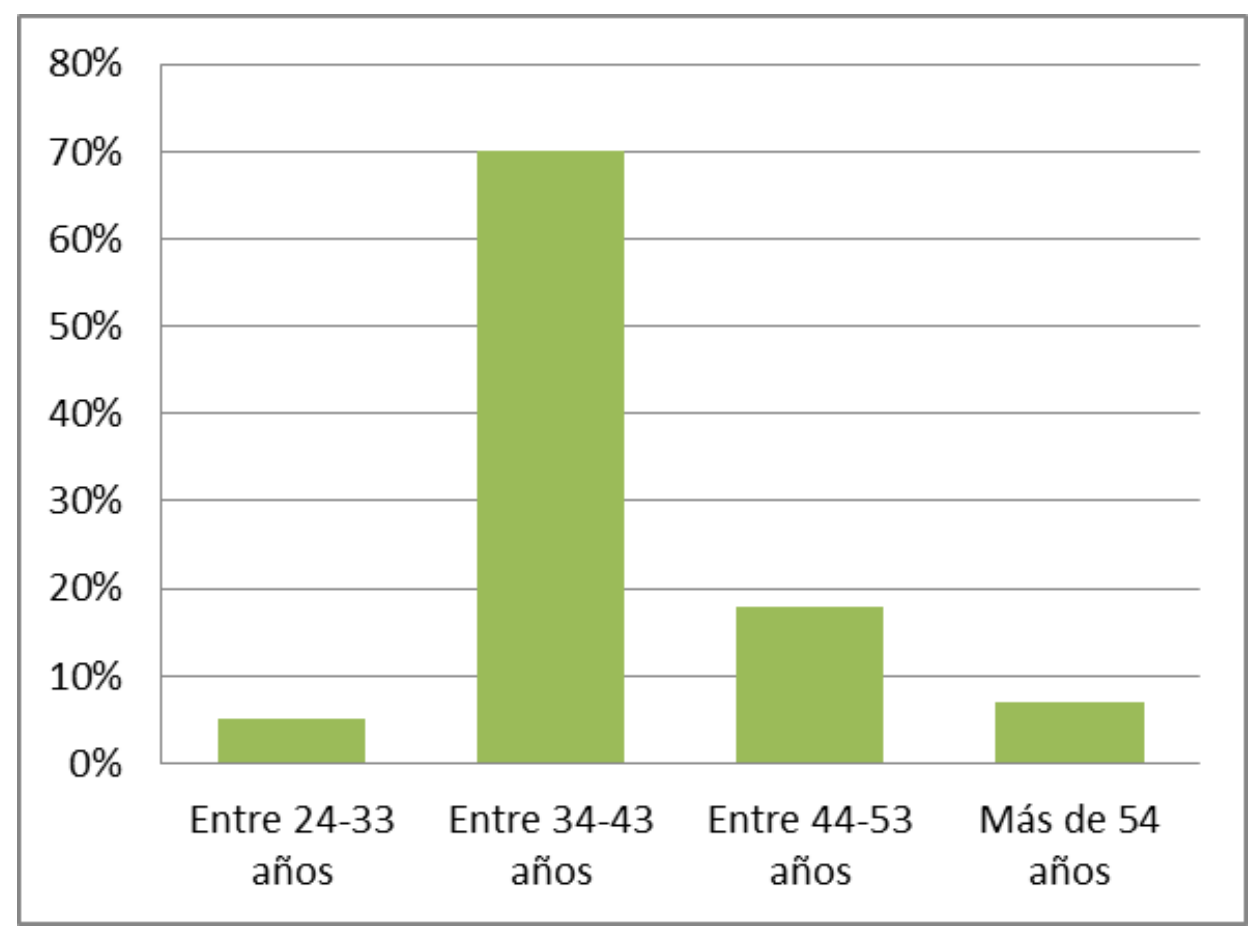

Fuente: elaboración propia a partir de los datos de la presente investigación. 
Gráfico 3. Formación de profesores

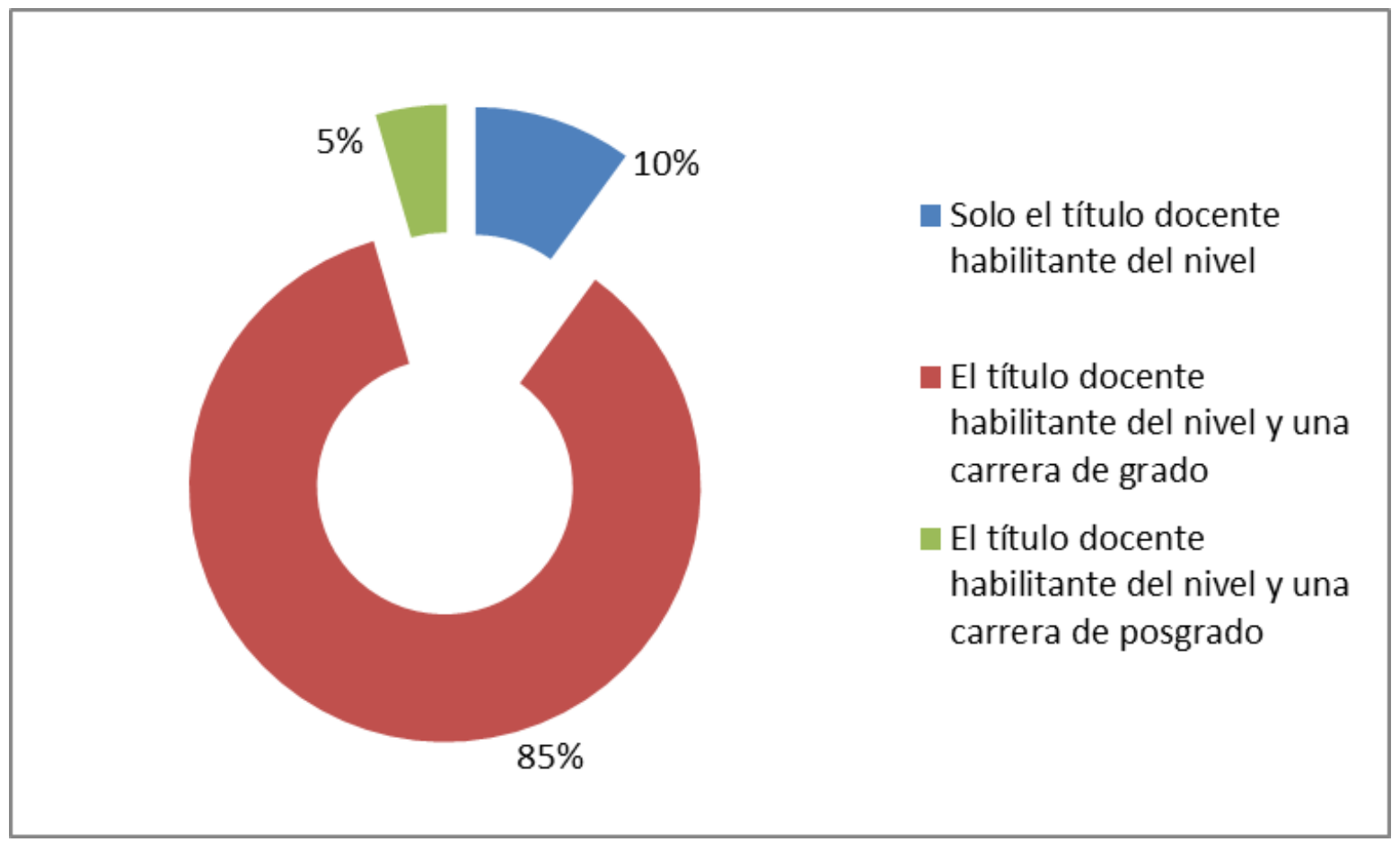

Fuente: elaboración propia a partir de los datos de la presente investigación.

\section{Instrumentos}

Para cumplir con los objetivos de esta investigación, se trabajó con fuentes primarias. Se llevaron a cabo entrevistas en profundidad a docentes y observaciones de clase. En el transcurso del trabajo de campo elaboramos notas de campo (Flick, 2014) para registrar los sentidos y percepciones surgidas.

En cada una de las instituciones se realizaron 10 observaciones de clase, conformando un total de 60 horas de observación. Se seleccionó esta técnica por su potencialidad para captar sentidos y adentrarnos en los aspectos centrales y recurrentes de la propuesta de enseñanza y formación en la interacción. A través de una rúbrica abierta, se efectuaron un total de 20 observaciones de clase, diez en cada escuela. El registro de observación fue abierto, con la intención de captar sentidos y singularidades, además de aspectos recurrentes. Los hallazgos se triangularon con las otras fuentes y se incluyeron en los resultados presentados.

Luego, se hicieron 20 entrevistas en profundidad a profesores. Se escogió este instrumento por su capacidad para explorar las percepciones con relación al uso de los medios digitales en la enseñanza. Se cumplimentaron 10 en cada una de las instituciones educativas.

Se elaboró un guion semi-estructurado configurado por nudos de sentido con los temas a dialogar. Su confección llevó diferentes etapas. Se inició con una exploración bibliográfica sobre el uso de los medios digitales en el espacio escolar para demarcar los tópicos a considerar en el instrumento. Se confeccionó un conjunto de interrogantes vinculadas a los tópicos. Luego, un grupo asesor externo, seleccionado por su pertinencia temática y accesibilidad, valoró el instrumento y realizó la evaluación de su consistencia. Tomando en cuento este dictamen, se elaboraron las 
reformas y se confeccionó la versión definitiva del guion (véase diagrama 1). El instrumento fue piloteado previamente con cinco docentes.

Diagrama 1. Selección del guión de entrevista

\section{Género:}

Edad:

Titulación:

Nivel educativo en el que ejerce:

Años de experiencia profesional:

¿Qué medios digitales utiliza en su práctica de enseñanza?

¿Qué formación recibió en el uso de los nuevos medios tecnológicos y su integración en el aula? ¿Qué valoración tiene de ella?

¿Cómo autoevalúa su manejo en herramientas digitales?

¿Qué conoce sobre el término Web 2.0?

¿Cuáles son sus usos en internet en el ámbito profesional y en el ámbito privado?

¿Qué disponibilidad en tiempo tiene para navegar en internet?

¿Qué tipo de conexión tiene?

¿Qué programas informáticos utiliza en sus prácticas de enseñanza?

¿Para que utiliza internet y las herramientas 2.0 en su actividad docente?

¿Cuáles son los recursos tecnológicos disponibles en su institución educativa?

¿Cómo integra los medios digitales en el ámbito docente?

¿Cómo considera la inversión económica y las políticas llevadas a cabo para la integración de los medios digitales en las aulas?

¿Qué relevancia tiene el uso de las redes sociales para la educación actual?

Fuente: elaboración propia a partir de los datos de la presente investigación

Las dimensiones y los aspectos que contemplan los instrumentos de recogida de datos son: la caracterización profesional (género, edad y título habilitante para ejercer el cargo), la formación en tecnologías (formación inicial y continua sobre el área, valoración personal del desempeño con los medios digitales, el entrena- miento en internet y la comprensión del vocablo web 2.0.), el acceso informático (tipo de conexión, uso de programas computacionales, uso de la web 2.0), destrezas pedagógicas con la web 2.0 (usos y propósitos de la inclusión de la web 2.0 en el ejercicio profesoral, recursos tecnológicos presentes en la escuela) y sentidos y percepciones (estimación de la inclusión de los medios tecnológicos en la enseñanza, estimación sobre las políticas educativas para la inclusión de los medios digitales en los salones de clase, estimación de la formación adquirida para incluir los medios digitales en el salón de clase y estimación del uso de las redes sociales para la formación en el siglo xxI).

\section{Consideraciones sobre el trabajo de campo}

El trabajo de campo se realizó en distintas fases. En una primera instancia se solicitó permiso a las autoridades educativas jurisdiccionales y distritales. En un segundo momento, se hizo una observación y entrevista institucional en cada una de las escuelas para explicar la propuesta al equipo directivo y requerir la colaboración de los profesores y estudiantes.

El trabajo de campo en cada caso implicó una serie de acuerdos previos con los docentes para llevar adelante la investigación. Tanto para la realización de las entrevistas y las observaciones de clase se trabajó con un consentimiento informado para el carácter voluntario del ejercicio y la confidencialidad de los datos. En sintonía con los lineamientos dados por el Consejo Nacional de Investigaciones Científicas y Técnicas (Resolución No 2857/ 2006), en el transcurso del estudio se tomaron las garantías éticas de rigor para conservar el carácter anónimo de los profesores y estudiantes que participen en la investigación, de forma informada, voluntaria, y confidencial. En este sentido, al solicitarles su participación, se entregaron a los sujetos Consentimientos Informados, en los que se explicaron brevemente los objetivos del plan de trabajo, y el carácter voluntario, de anonimato y confidencialidad en la administración de la información recogida. En la exposición de los 
resultados, tomamos los resguardos necesarios para preservar la identidad de los sujetos y las instituciones que participaron de la investigación.

Luego de tres meses se realizaron en las escuelas las entrevistas a docentes, las observaciones de clase y los documentos.

\section{Estrategias de análisis}

El estudio de la información se realizó según el propósito de la investigación, partiendo de la información obtenida de cada actor participante. Las entrevistas y observaciones de clase fueron analizadas de acuerdo con el denominado ordenamiento conceptual (Piovani, 2018). Este proceso de organización de los datos se realizó en función de las propiedades y dimensiones de las categorías. Para la sistematización se utilizó el Atlas Ti 8 como herramienta informática, un software especialmente desarrollado para el tratamiento de datos cualitativos. Una vez elaboradas las familias de categorías, se generaron hipótesis interpretativas de mayor nivel de abstracción respecto de la información empírica obtenida. Un aspecto relevante es que el análisis se ubicó en distintas etapas en la extensión de la investigación.

Si bien esta investigación obedece al enfoque cualitativo, para facilitar la visualización de los hallazgos se optó por la organización en porcentajes. El uso doméstico y pedagógico que los profesores realizan de la web 2.0 es concebido como una estructura de significados. A través de estos análisis, se buscó comprender los procesos sociales involucrados, profundizando su especificidad a partir del descubrimiento del significado que dicho hecho tiene para sus "actores".

Señalamos también que hicimos un trabajo de triangulación entre los análisis provenientes de ambas fuentes, permitiendo lograr una mayor fiabilidad de los datos y reduciendo así los sesgos propios. La triangulación se constituye en aporte para construir sentidos sobre los datos y como forma de validación de los análisis realizados desde una perspectiva comprensiva.

\section{Resultados}

\section{La formación tecnológica de los profesores de nivel secundario}

En las últimas décadas la problemática de la formación docente se ha constituido en un foco privilegiado e insoslayable de cualquier estrategia de cambio y de transformación educativa. Las políticas de formación docente se encuentran en la mayoría de las iniciativas de mejora de los sistemas educativos, tanto en Argentina como en el contexto regional e internacional. Se destaca la importancia de generar conocimiento, para preparar docentes capaces de saber y poder enseñar en los escenarios educativos del presente.

A pesar de que la mayoría de los docentes consultados $(85 \%)$ expresan que no recibieron capacitación específica sobre el uso de los medios digitales en los espacios de formación docente, todos manifiestan dominar los programas y recursos tecnológicos, y tener capacidades para usar internet, los buscadores y el correo electrónico (véase gráfico 4). Solo un $15 \%$ admite tener capacidad para manejar las plataformas de teleformación (Moodle, WebCT, Illias o Elvira, entre otras).

Gráfico 4. Formación en medios digitales de los profesores de nivel secundario

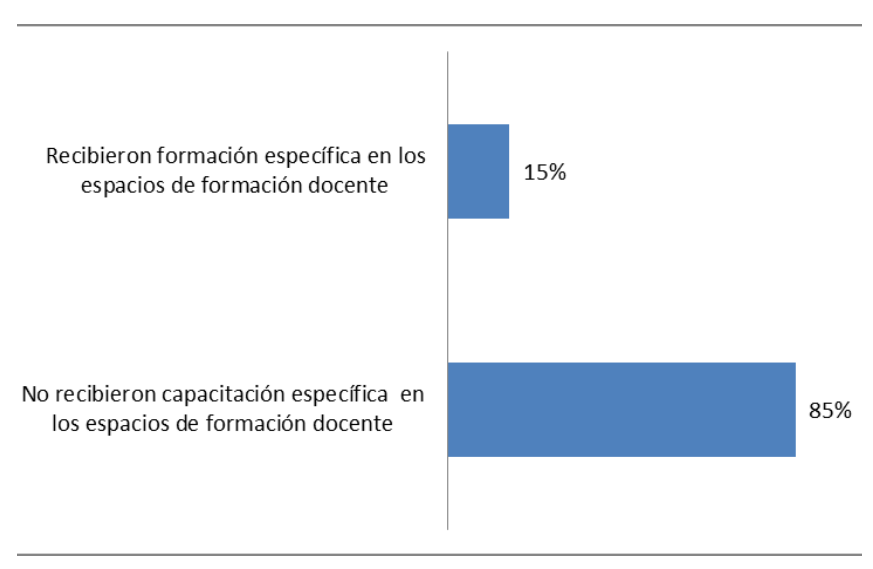

Fuente: elaboración propia a partir de los datos de la presente investigación. 
Los profesores señalan que han sido autodidactas en el uso de internet, manifiestan que ni los espacios de formación inicial ni los espacios de formación permanente han facilitado este aprendizaje. Finalmente, más de la mayor parte de los docentes afirman que no comprenden el vocablo web 2.0, a pesar de que pueden manejar algunas herramientas.

\section{Perfil informático del profesorado}

La totalidad de los docentes entrevistados tiene una conexión a internet en su hogar, con un tipo de conexión ADSL. Así también disponen de un dispositivo móvil inteligente con un tipo de conexión $4 \mathrm{G}$. Respecto al uso de programas computacionales, enfa- tizan que en el contexto doméstico hacen uso de los procesadores de textos como herramientas para resolver experiencias en ese ámbito. Con respecto al uso de otros programas, aluden a los referidos a las presentaciones (ejemplo: PowerPoint) y hojas de cálculo (Excel). El buscador más usado es el de Google (90 \%), y la elección favorita (70 \%). Las actividades que llevan a cabo en internet son: revisar el correo electrónico, leer noticias y acceder a redes sociales. Es relevante mencionar que todos los profesores entrevistados tienen un correo electrónico gratuito. Finalmente, uno de los espacios de comunicación más usados por estos profesores son las redes sociales, $y$ Facebook es la preferida (75\%) (véase gráfico 5).

Gráfico 5. Espacios de comunicación más usados

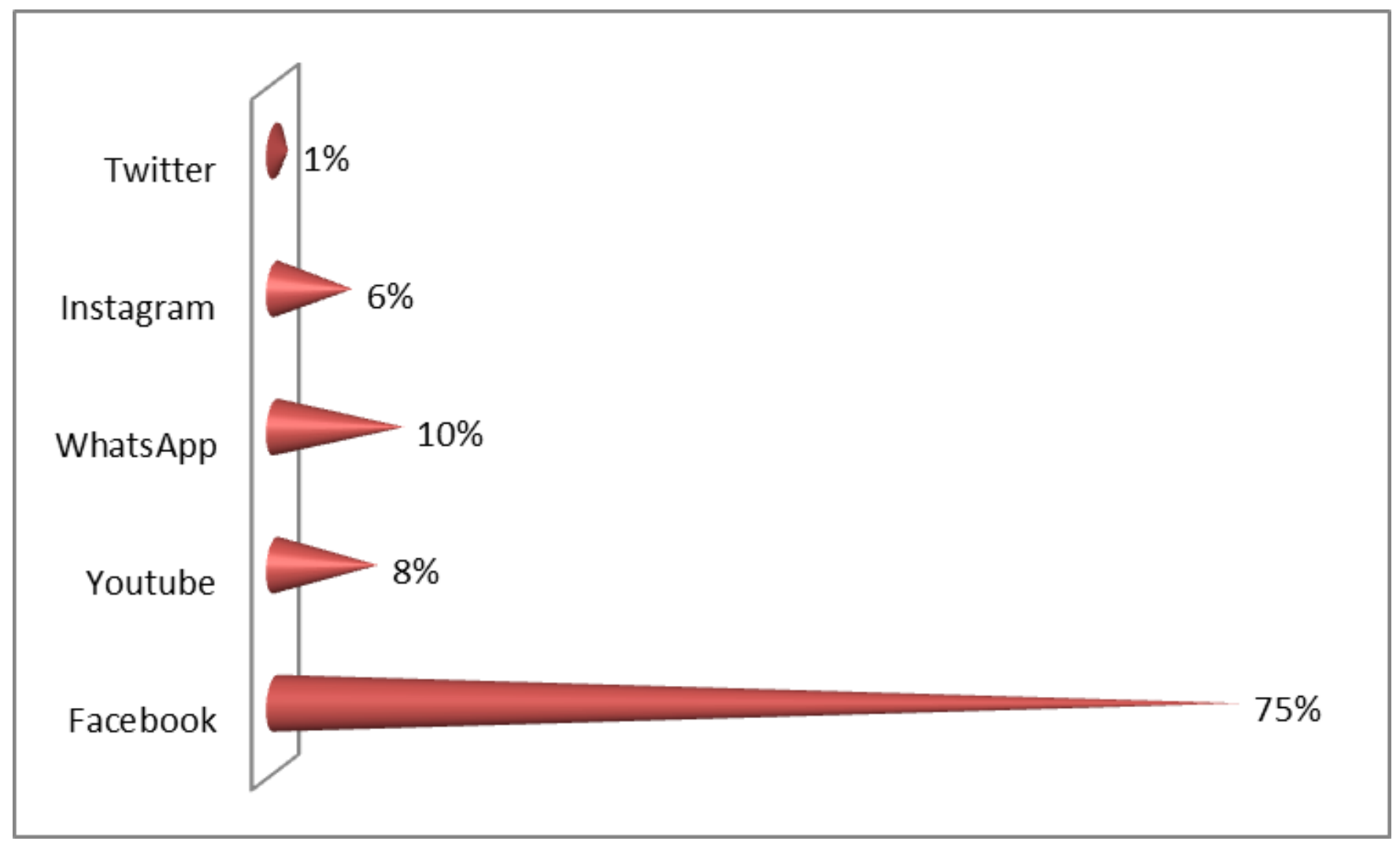

Fuente: elaboración propia a partir de los datos de la presente investigación. 


\section{Prácticas pedagógicas con la web 2.0}

La totalidad de los profesores indica que disponen de ordenadores en su lugar de trabajo. Se trata de equipos netbooks de uso compartido con otros compañeros, en una proporción de 60 ordenadores para una población de escolar de 200 individuos. Estos fueron proporcionados por los planes de inclusión digital mencionados en la introducción del presente artículo.

Un aspecto relevante sobre los usos y propósitos de la inclusión de la web 2.0, es que prefieren utilizan el correo electrónico y el WhatsApp para el contacto entre colegas docentes y directivos, y desmerecen estas herramientas en la relación con los estudiantes. Excepto que se despliegue alguna situación de salud que amerite que el estudiante vea afectada su regular asistencia a la escuela. El argumento de esta distinción refiere a que se ve desdibujada la relación pedagógica profesor-estudiante, pueden suscitarse conflictos y problemas de comunicación informal, e implica una sobrecarga de su labor docente.

Esto conduce a que tampoco se utilicen estas herramientas de la web 2.0 en su labor docente dentro del aula. Solamente se solicita la búsqueda de información como tarea a realizar por fuera de la escuela, donde la recomendación es la utilización del buscador Google.

Se destaca que los participantes de la muestra manifiestan el uso de dichas herramientas como recurso para intervenir en los proyectos educativos y como medio para la mejora en la enseńanza.

Solo el $15 \%$ de los profesores revela que se siente competente para la inclusión de la web 2.0 en su ejercicio profesoral de forma sistemática (véase gráfico 6). Y la totalidad de los profesores manifiestan que su escuela no proporciona las condiciones de infraestructura para el uso de la misma.

Gráfico 6. Percepción sobre la inclusión de la web 2.0 de forma sistemática

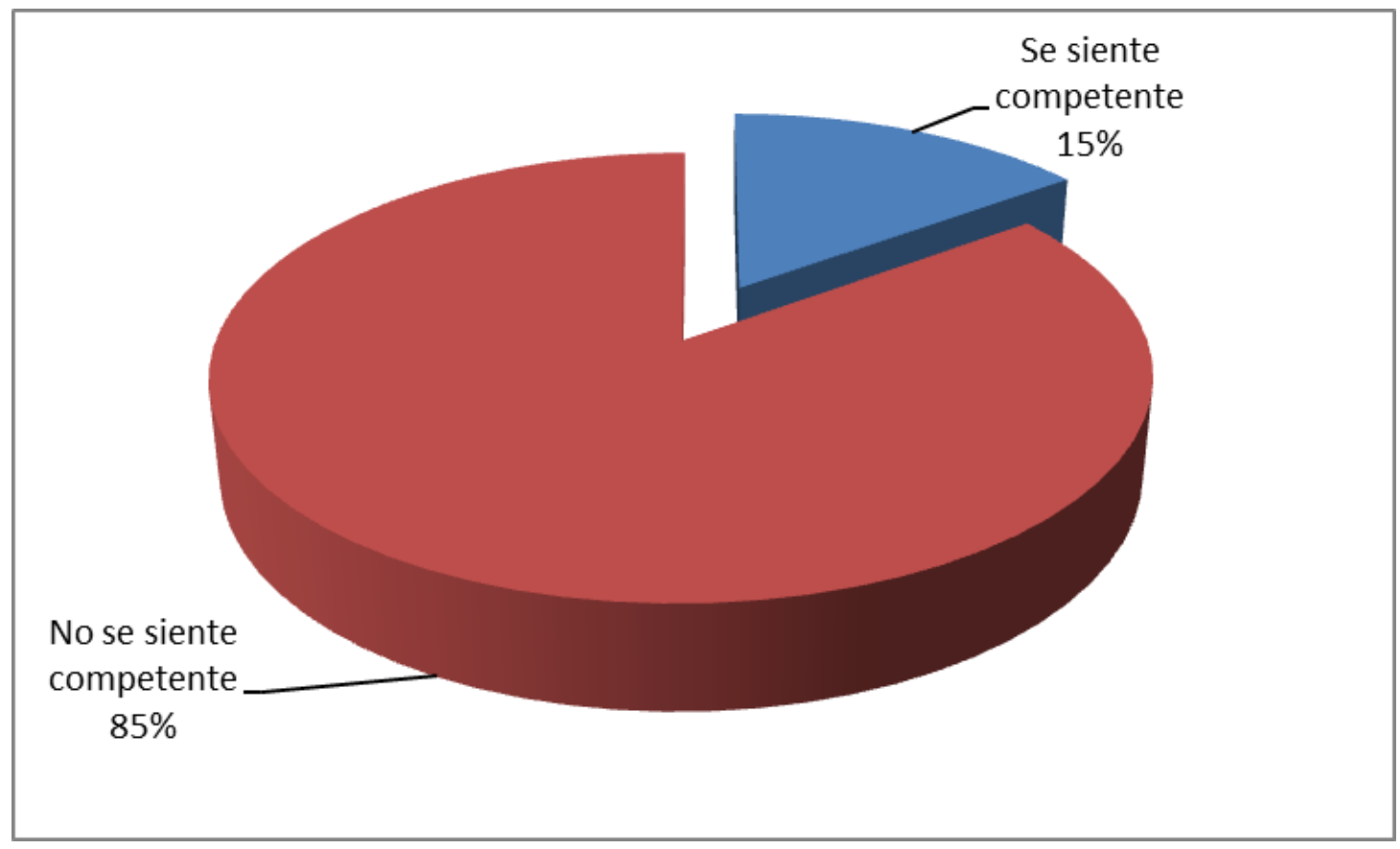

Fuente: elaboración propia a partir de los datos de la presente investigación. 


\section{Sentidos y percepciones}

La totalidad de los profesores estima imprescindible la inclusión de los medios digitales en las estrategias de enseńanza, argumentando que son herramientas propias de la sociedad actual, posibilitan el uso de recursos multimedia, aumenta la motivación del estudiantado, están en permanente transformación y se les solicitan a los estudiantes como requisitos para optar por un trabajo. Sin embargo, aducen que en su contexto educativo es difícil implementarlo dada la falta de capacitación que tienen sobre el tema, y que no conocen los usos pedagógicos de estas herramientas.

Respecto a la valoración de la inclusión de los medios digitales en las clases, la totalidad de los profesores estima que los medios digitales son un recurso complementario en la estrategia de enseñanza y considera que es un recurso y no una estrategia de enseñanza en sí misma.

En la comunicación entre colegas docentes destacan las ventajas de la comunicación no mediada por medios digitales, directa y personal. En tanto los canales que se abren por mail y por WhatsApp no favorecen el intercambio para el trabajo grupal y suelen ocasionar conflictos vinculares (especialmente el WhatsApp).

Por último, la totalidad de la muestra afirma que podría mejorarse el soporte brindado por la escuela. Esgrimiendo que, entre otros aspectos, es lento el acceso a los recursos, y cuando se brindan no son los solicitados o suficientes. Así, también identificamos que no hay acuerdo en el acondicionamiento de la inversión económica.

\section{Conclusiones}

Existen variados proyectos pedagógicos que se centran en la integración de las herramientas 2.0 en la escuela secundaria argentina (Maggio, 2019; Saez, 2019b). Sin embargo, los profesores son los que poseen la decisión clave de incluir los medios digitales en sus estrategias de enseńanza. En la sociedad del siglo XXI son cuantiosas las experiencias escolares en las que el profesor debe formar mediáticamente a un estudiantado. Así, se hace imprescindible recuperar las voces de los educadores y detenerse a analizarlas ante el desafío de la integración de los nuevos medios a las estrategias de enseńanza (Escudero y Rodríguez, 2013).

En este artículo se ha caracterizado el uso doméstico y pedagógico que los profesores realizan de la web 2.0. En continuidad con las investigaciones de las pedagogías críticas (Jenkins et al., 2019; Dussel y Trujillo, 2018; Southwell, 2019; Tyner, 2014; Gutiérrez y Torrego, 2018), en este trabajo observamos cómo lo digital interpela la delimitación estática de los espacios educativos, al mismo tiempo que nos convoca a analizar críticamente el concepto de alfabetización.

Tras el análisis de la muestra se identifica que los profesores tienen destreza en las herramientas de comunicación asíncrona tradicional, como por ejemplo el correo electrónico, y tienen poco manejo de las herramientas de nueva generación o 2.0. Los educadores se manifiestan como usuarios a nivel doméstico de herramientas 2.0, y para el uso personal se reconocen como usuarios frecuentes en las redes sociales. Estos datos se agravan al focalizarnos en los usos pedagógicos de los medios digitales.

Resulta alarmante que no hagan uso de las herramientas 2.0 en su actividad pedagógica. Esto tiene correlato con los resultados obtenidos por Padilla (2018) y Maenza y Sgreccia (2011), que muestran que los profesores no se sienten capacitados para responder a las exigencias de su estudiantado ni de la cultura digital, y no están formados para transformar esta realidad.

En cuanto al acceso de los recursos tecnológicos, los educadores cuentan con las herramientas en sus casas - computadoras, celulares inteligentes, etc.sin embargo, no sucede esto en las escuelas, en tanto en la mayor parte de las veces comparten ordenadores con otros profesores o apelan a usar sus celulares inteligentes de uso personal. Esta evidencia tiene relación con que realizan un uso más doméstico que pedagógico de las herramientas. Surge como hipótesis interpretativa que la falta de recursos suficientes 
imposibilita un mayor uso de la web 2.0 en el espacio escolar.

Ahora bien, los profesores reconocen los beneficios de incluir la web 2.0 (motivación del estudiantado, recurso novedoso, fortalece la estrategia de enseñanza), pero su utilización es muy restrictiva todavía y varias de las experiencias se orientan a imitar las actividades que ya se realizaban con otros recursos, como la búsqueda de información. Las tecnologías funcionan y se amplifican en el marco de prácticas culturales más amplias, por lo que no son neutrales y pueden tanto potenciar como obstaculizar las prácticas educativas (Buckingham, 2019). Lo determinante no es qué tan nueva es la tecnología implementada en la escuela, sino que los "viejos" medios digitales se redefinen a la luz de la incorporación de los "nuevos", a modo de "transición" (Dussel y Trujillo, 2018).

Los resultados arrojados identifican una relevante debilidad en la formación, y los entrevistados afirman no reconocerse formados para usar las herramientas 2.0 con un sentido pedagógico; observan carencias en la educación recibida sobre el uso pedagógico de la web 2.0.

Ahora bien, en Argentina se entregaron computadoras portátiles a estudiantes y docentes del sistema público y de gestión social, pero el esfuerzo económico no tuvo los resultados esperados, entre otras causas por la carencia en la capacitación pedagógica de los educadores en los medios digitales. En la literatura internacional, investigaciones del campo de la tecnología educativa observan cómo las exigencias para la incorporación de los medios digitales y las experiencias de innovación modifican tanto las prácticas pedagógicas como las institucionales, mayormente desde una mirada tecnicisista o eficientista, relegando su incorporación al "voluntarismo" de los docentes (Montero y Gewerc, 2018). Por su parte, en el ámbito argentino, las indagaciones plantean la necesidad de una política que tienda a una inclusión genuina, por convicción de los docentes, que reconozca el valor de los medios digitales y los nuevos entornos tecnológicos en los campos discipli- nares y en la formación, más allá de la incorporación por imposición del Estado o las modas circunstanciales (Maggio, 2019). En la misma línea se debate el concepto de nuevos medios, ya que el "valor pedagógico" de las propuestas no radican en lo novedoso de las tecnologías incorporadas, sino en el grado de apropiación y construcción del conocimiento que incentivan, propios de la cultura digital (Litwin, 2009). En este carril, consideramos que la Educación Mediática no remite a una formación técnica solamente, sino que contiene el desarrollo de una mirada inteligente. El reto no radica tanto en la inclusión de las nuevas herramientas, aunque son condición de posibilidad, sino en la posibilidad de generar situaciones que permitan construir un posicionamiento crítico. La sociedad del siglo xxi está en los salones de clase, el desafío es cómo habilitar experiencias para pensar y comprender la vida social (Buckingham, 2019).

La cuestión de los nuevos medios ha cobrado creciente importancia en relación con la educación, y la ley de educación argentina vigente destaca la relevancia de habilitar ambientes educativos para la integración de las nuevas tecnologías de la información y la comunicación. Esto representa un desafío pedagógico en la construcción de conocimiento, ya que el aumento de la escolarización coexiste con múltiples brechas socioeconómicas y socioculturales. Las desigualdades en el acceso a los medios tecnológicos no solo crean una brecha digital, sino que generan, especialmente, una brecha social.

Con estos resultados se concluye que los profesores de la escuela secundaria argentina necesitan de una educación mediática que los habilite en un primer momento a manejar de forma idónea las herramientas 2.0 y de sus usos pedagógicos, con el propósito de que se genere una transformación en las estrategias de enseñanza que la sociedad del siglo XXI requiere.

Las potenciales razones que evidencian la falta de educación mediática en el profesorado pueden hallarse en tres núcleos. En primer lugar, los recursos informáticos a los que tienen acceso los educadores en las escuelas secundarias son escasos. En segundo 
término, carecen de capacitación suficiente, provocando una innegable incertidumbre para integrar las herramientas con un fin pedagógico. Y, finalmente, se requiere una transformación del piso tecnológico y recursos.

Esta investigación aportó resultados sobre las condiciones y transformaciones del trabajo docente en la era digital, relevando sentidos y prácticas sobre el uso de los medios tecnológicos en el nivel secundario argentino. Este estudio se formula para aportar al conocimiento del campo de la educación y al de la formación docente. Se traza como un tópico relevante en perspectiva regional.

En futuras investigaciones indagaremos los usos genuinos y significativos de los recursos digitales en el marco de las prácticas docentes, que impliquen nuevas habilidades cognitivas y multiplicidad de formas de representación lingüísticas y comunicacionales.

El derecho a la educación y el derecho a la comunicación constituyen una puerta de acceso a otros derechos humanos. Promover una educación mediática de calidad es una acción estratégica para consolidar una formación de calidad. Se trata de generar nuevos parámetros en la educación de la mirada en pos de fortalecer el alcance del rol docente.

\section{Referencias}

Buckingham, D. (2019). Teaching media in a 'posttruth' age: fakenews, mediabiasand thechallenge far media/digital literacy education / La enseñanza mediática en la era de la posverdad: fake news, sesgo mediático y el reto para la educación en materia de alfabetización mediática y digital. Cultura y Educación, 31(2), 213-231, Doi: 10.1080/11356405.2019.1603814

Delgado, V., Casado, R., y Lezcano, F. (2016). La formación permanente del profesorado universitario en el EEES: Un estudio en la Universidad de Burgos. @tic. revistad'innovació educativa.17, 30-40. Recuperado de http://doi.org/10.7203/ attic. 17.9105
Dussel, I. y Quevedo, L.A. (2010). Educación y Nuevas Tecnologias: Los desafíos pedagógicos ante el Mundo digital. Buenos Aires: Santillana.

Dussel, I. y Trujillo, B. F. (2018). ¿Nuevas formas de enseñar y aprender? Las posibilidades en conflicto de las tecnologías digitales en la escuela. Perfiles Educativos, 40(Especial), 142-178. Recuperado de https://doi. org/10.22201/iisue.24486167e.2018.Especial.59182

Escudero, J.M., González, M. y Rodríguez, M. (2013). La mejora equitativa de la educación y la formación del profesorado. Multidisciplinary Journal of Educational research, 3(2), 206-234.

Ferrés, J. y Piscitelli, A. (2012). La competencia mediática: propuesta articulada de dimensiones e indicadores. Comunicar, 19(38), 75-82. Recuperado de https://www.redalyc. org/articulo.oa? id=158/15823083010

Flick, U. (2014). La gestión de la calidad en investigación educativa. Madrid: Morata.

Gutiérrez, A. y Torrego, A. (2018). Educación Mediática y su Didáctica. Una Propuesta para la Formación del Profesorado en TIC y Medios. Revista Interuniversitaria de Formación del Profesorado, 32(1), 15-27. Recuperado de: https://www.redalyc.org/articulo. oa?id=274/27454937002

Hernández, R., Fernández, C. y Baptista, P. (2010). Metodología de la investigación (5a ed). México, D.F: McGraw-Hill.

Jenkins, H., Saad, E., Fung, A. y Bosch, A. (2019). Making Media: production, Practices, and Professions. Países Bajos: Amsterdam University Press.

Kaplan, C.V. y Piovani, J.I. (2018). Trayectorias y capitales socioeducativos. En J.I. Piovani, y A. Salvia (eds.). La argentina en el siglo XXI. Cómo somos, vivimos y convivimos en una sociedad desigual, (pp. 221-263). Buenos Aires: Siglo XXI.

Kerbrat, C. (1980). La enunciación. De la subjetividad en el lenguaje. Buenos Aires: Hachette. 
Litwin, E. (2009) "La tecnología educativa en el debate didáctico contemporáneo". En: Tecnologias educativas en tiempos de Internet. Buenos Aires: Amorrortu.

Maenza, R. y Sgreccia, N. (2011). Uso de herramientas web 2.0 con futuros docentes. Profesorado. Revista de curriculum y formación del profesorado, 15(3). Recuperado de https:// www.ugr.es/ - recfpro/rev153COL5.pdf

Maggio, M. (2019) La reinvención colectiva de las prácticas de la enseńanza como desafío de la educación latinoamericana contemporánea. Cuadernos de pedagogía, 500(0), 146-150.

Martín Barbero, J. (2019) Mapas nocturnos y mediaciones diurnas. Philosophical Readings, 11(3), 193-198. Doi: 10.5281/zenodo.3560373

Montero L. y Gewerc, A. (2018) La profesión docente en la sociedad del conocimiento. Una mirada a través de la revisión de investigaciones de los últimos 10 años. RED. Revista de Educación a Distancia, 56(0), 1-22. Recuperado de http:// dx.doi.org/10.6018/red/56/3

Orozco, G. (2018) La Múltiple Audienciación de las Sociedades Contemporáneas: Desafíos para su Investigación. Anuario Electrónico de Estudios en Comunicación Social "Disertaciones", 11(1). Recuperado de https://doi.org/10.12804/ revistas.urosario.edu.co/disertaciones/a.6274

Padilla, S. (2018). Usos y actitudes de los formadores de docentes ante las TIC. Entre lo recomendable y la realidad de las aulas. Apertura (Guadalajara, Jal.), 10(1), 132-148. Recuperado de https:// dx.doi.org/10.18381/ap.v10n1.1107

Piovani, J.I. (2018). Manual de Metodología de las ciencias sociales. Buenos Aires: Siglo Veintiuno.

Saez, V. (2019a) La Educación Mediática en la Escuela Secundaria desde los diseños curriculares. Entramado (Universidad Libre de Colombia), 15(2), 148-159. Recuperado de http://dx.doi.org/10.18041/1900-3803/entramado.2.5629Entramado
Saez, V. (2019b). Los rasgos de la educación mediática en el nivel secundario: un estudio del caso argentino. Actualidades Pedagógicas, (74), 1-17. Recuperado de https://doi.org/10.19052/ ap.vol1.iss 74.5

Saez V. y Richter N. C. (2019). La educación mediática como campo interdisciplinario. En V. Saez (coord). Educación de la Mirada. Experiencias, reflexiones y desafíos pedagógicos de la formación en Medios, Comunicación y Tecnologías en Argentina. (pp. 6-19). Buenos Aires: Facultad de Ciencias Sociales de la Universidad de Buenos Aires. Recuperado de http:// educaciondelamirada.com/investigacion/ educacion-de-la-mirada-experiencias-reflexiones-y-desafios-pedagogicos-de-la-formacion-en-medios-comunicacion-y-tecnologias-en-argentina/

Sirvent, M.T., Monteverde, A.C., Clerici, C., Peruzzo, L.M. y Agulló, M. (2016). Algunas reflexiones acerca del proceso de formación en investigación de estudiantes de grado y posgrado. $V$ Encuentro de Metodología de las Ciencias Sociales "ELMECS". Mendoza, FCPYS-UNCUYO, 16 al 18 de noviembre de 2016. Recuperado de http://elmecs.fahce.unlp.edu.ar/v-elmecs/ actas-2016/Sirvent.pdf

Southwell, M. (2019). Vínculos intergeneracionales y formas de reconocimiento en la escuela secundaria: algunas notas conceptuales. Revista Ensambles, 8(0), 65-85

Tyner, K. (2014). Literacy in a Digital World: Teaching and Learning in the Age of Information. New York: Routledge. Recuepardo de https://doi.org/10.4324/9781410601971 